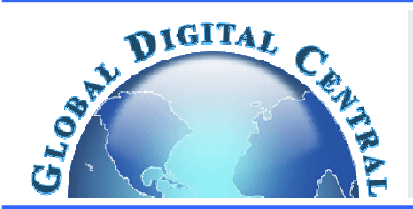

Frontiers in Heat and Mass Transfer

Available at www.ThermalFluidsCentral.org

\title{
INFLUENCE OF VARIABLE THERMAL CONDUCTIVITY ON MHD CASSON FLUID FLOW OVER A STRETCHING SHEET WITH VISCOUS DISSIPATION, SORET AND DUFOUR EFFECTS
}

\author{
B. Venkateswarlu ${ }^{a}$, P.V. Satya Narayana ${ }^{\text {b, * }}$ \\ ${ }^{a}$ Department of Mathematics, Madanapalle Institute of Technology \& Science, Madanapalle, A.P., India \\ ${ }^{b}$ Department of Mathematics, SAS, VIT University, Vellore - 632014, T.N., India
}

\begin{abstract}
This paper studies the Soret and Dufour effects on MHD flow of a Casson fluid past a stretching sheet in the presence of chemical reaction, viscous dissipation and variable thermal conductivity. The fluid is taken to be electrically conducting and the flow is induced by a stretching surface. The governing partial differential equations are transformed into non-linear ordinary differential equations using similarity transformations. The resulting equations are then solved numerically by shooting method. The impact of various stimulating parameters on the flow, heat and mass transfer characteristics are analyzed and discussed in detail through graphs. It is observed that the thermal conductivity parameter has a lot of control on heat and mass transfer rates. A comparison with previously published work is accomplished and the outcomes are found to be in excellent agreement.

Keywords: Casson fluid, Soret and Dufour numbers, Viscous dissipation, Variable thermal conductivity, Chemical reaction
\end{abstract}

\section{INTRODUCTION}

The boundary layer flows of non-Newtonian fluid persuaded by a stretching surface are encountered in several engineering processes. The review of non-Newtonian flows with heat transfer is of prodigious significance in many manufacturing processes such as in glass sheets blowing, food processing, hot rolling and paper production etc, (Olajuwon, 2013; Swati Mukhopadhyay et al., 2012; Bird et al., 1983). In view of various rheological properties of non-Newtonian fluids in nature, there is no specific constitutive connection between stress and rate of strain by which all the non-Newtonian fluids can be observed. Therefore, several non-Newtonian fluid models (Power-Law Fluid, Prandtl-Eyring Model, Bingham Fluids, Powell-Eyring Model and Maxwell fluid model) have been suggested. Among these, Casson fluid is the simplest model for the rheological effects of viscoelastic fluids. Initially, the Casson model was introduced by Casson (1959). Later, Bird et al. (1983) reported that Casson fluid model exhibits shear thinning characteristics, yield stress and higher viscosity. In addition to this, it estimates practically well the rheological performance of other liquids such as syrups and cosmetics etc. The Casson fluid flow over a stretching sheet is significant in the field of metallurgy and chemical engineering. Mustafa et al. (2011) presented an unsteady boundary layer flow of a Casson fluid due to an impulsively started moving flat plate. Bhattacharya et al. (2013) studied the MHD boundary layer flow of Casson fluid over a permeable stretching/shrinking sheet. Nadeem et al. (2013) investigated the Casson fluid flow in two lateral directions past a porous linear stretching sheet in the presence of magnetic field. Mukhopadhyay (2013) analyzed the Casson fluid flow and heat transfer over a nonlinearly stretching surface. Shehzad et al. (2014) studied the thermophoresis particle deposition in mixed convection threedimensional radiative flow of an Oldroyd-B fluid. Satya Narayana et al. (2015) presented the combined effects of heat and mass transfer in Jeffrey fluid over a stretching sheet with power law form of temperature and concentration. Chemical reaction and radiation absorption effects on the flow and heat transfer of a nanofluid in a rotating system have been presented by Venkateswarlu and Satya Narayana (2015). Kataria and Patel (2016) have investigated the radiation and chemical reaction effects on MHD Casson fluid flow past an oscillating vertical plate embedded in a porous medium. Satya Narayana and Venkateswarlu (2016) have studied heat and mass transfer on MHD nanofluid flow past a vertical porous plate in a rotating system. Some pertinent surveys which have been carried out recently are presented in Refs. (Hayat et al. 2012; Raju et al. 2014; Ahmed et al. 2014; Sheikholeslami and Rashidi, 2015; Imran Ullah et al., 2016; Raju et al., 2016).

All the above mentioned articles deal with the constant physical properties of the fluid. But in reality, most of the practical situations claims for physical properties of the fluids vary significantly with temperature. It is reported (Miao and Massoudi, 2015; Anjali Devi and Prakash, 2015) that the thermal conductivity is inclined to vary with a temperature gradient, especially in the boundary layer region. The variable thermal conductivity plays a vital role in many engineering and industrial applications. In liquid metals, it has been observed that, the thermal conductivity varies with temperature in an approximately linear manner in the range from 0 to $400 \mathrm{~F}$ (see Kays, 1966). Variable thermal conductivity in convective heat transfer problems may be observed in heat exchangers and cooling systems of electronic devices in which the changes of temperature and hence the variations of thermal conductivity are huge Talukdar and Mishra, (2002). The concept of firm thermal conductivity in simulating such cases may effect in large inaccuracy (see Ref. Saravanan and Kandaswamy (2000)). The viscosity of water decreases by about $240 \%$ when the temperature increases from $10^{0} \mathrm{C}$ $(\mu=0.0131 \mathrm{~g} \mathrm{~cm}-1 \mathrm{~s}-1)$ to $50{ }^{0} \mathrm{C}(\mu=0.00548 \mathrm{~g} \mathrm{~cm}-1 \mathrm{~s}-1)$ (see Ref. Abd El-Aziz (2007)). Abel et al. (2009) studied the effects of thermal buoyancy and variable thermal conductivity on the MHD flow and heat transfer in a power-law fluid past a vertical stretching sheet in the presence of a non-uniform heat source. Animasaun (2015) analyzed the effects of thermophoresis, variable viscosity and thermal

\footnotetext{
*Corresponding Author. Email: pvsatya8@yahoo.co.in.
} 
conductivity on free convective heat and mass transfer of non-darcian MHD dissipative Casson fluid flow with suction and $\mathrm{n}^{\mathrm{th}}$ order of chemical reaction. More recently, Animasaun et al. (2016) studied Casson fluid flow with variable thermo-physical property along exponentially stretching sheet with suction and exponentially decaying internal heat generation using the homotopy analysis method. Pramanik (2014) analyzed the boundary layer flow of a non-Newtonian fluid accompanied by heat transfer toward an exponentially stretching surface with suction or blowing at the surface. Bhattacharyya et al. (2016) presented an exact solution for thermal boundary layer in Casson fluid flow over permeable shrinking sheet with variable wall temperature and thermal radiation. Many authors (Satya Narayana et al. 2013a; 2013b; 2015a; 2015b; Venkateswarlu and Satya Narayana, 2015; Vijayalakshmi and Srinivas 2016; Kamalakar et al., 2016) have investigated heat and transfer flow characteristic of Newtonian/nonNewtonian fluids over different geometries.

To the best of authors' knowledge, no attempt has been made for the Casson fluid flow past a stretching sheet with variable thermal conductivity, thermo diffusion and diffusion thermo effects. The problem which is discussed here is an important one that arises in many practical circumstances such as polymer extrusion process. Similarity solutions are obtained and the reduced ordinary differential equations are solved numerically using shooting technique with Runge-Kutta integration scheme, which is more economical from the computational point of view. The effects of different flow parameters encountered in the equations are studied with the help of their graphical representations.

\section{MATHEMATICAL FORMULATION OF THE PROBLEM}

We consider the steady MHD laminar boundary-layer flow heat and mass transfer of an incompressible, electrically conducting Casson fluid past a stretching surface. The surface is stretched in its own plane with a velocity proportional to its distance from the fixed origin $x=0$ (see Fig.1). The motion in an incompressible fluid is induced because of the stretching property. This occurs in view of the elastic properties of the surface parallel to the $x$-axis through equal and opposite forces when the origin is fixed. It is assumed that the fluid is electrically conducting in the presence of a constant magnetic field $B_{0}$ which is exerted in the transverse direction to the surface. The induced magnetic field is negligible due to small magnetic Reynolds numbers. The rheological equation of extra stress tensor $(\boldsymbol{\tau})$ for an isotropic and incompressible flow of a Casson fluid can be written as

$$
\tau_{i j}=\left\{\begin{array}{ll}
2\left(\mu_{B}+\frac{p_{y}}{\sqrt{2 \pi}}\right) e_{i j} & \pi>\pi_{c} \\
2\left(\mu_{B}+\frac{p_{y}}{\sqrt{2 \pi_{c}}}\right) e_{i j} & \pi<\pi_{c}
\end{array}\right\}
$$

where $P_{y}=\frac{\mu_{B \sqrt{2 \pi}}}{\beta}$ is the yield stress of the fluid

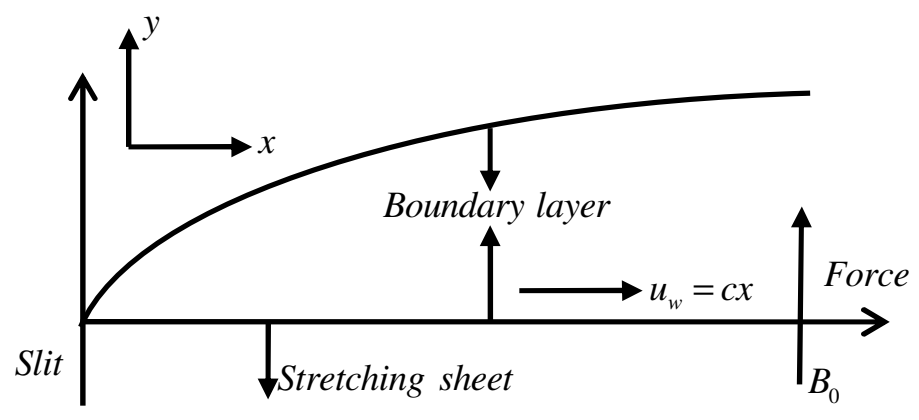

Fig. 1 Schematic diagram of the physical model
Under the usual boundary layer approximation, the governing equations describing the conservation of mass, momentum, energy and concentration in the presence of variable thermal conductivity are governed by the following equations (see Refs. (Hayat et al. 2012; Mustafa et al. 2012).

$$
\begin{aligned}
& \frac{\partial u}{\partial x}+\frac{\partial v}{\partial y}=0 \\
& u \frac{\partial u}{\partial x}+v \frac{\partial u}{\partial y}=v\left(1+\frac{1}{\beta}\right) \frac{\partial^{2} u}{\partial y^{2}}-\frac{\sigma B_{0}^{2}}{\rho} u \\
& u \frac{\partial T}{\partial x}+v \frac{\partial T}{\partial y}=\frac{1}{\rho C_{P}} \frac{\partial}{\partial y}\left\{K(T) \frac{\partial T}{\partial y}\right\}+\frac{v}{C_{P}}\left(1+\frac{1}{\beta}\right)\left(\frac{\partial u}{\partial y}\right)^{2}+\frac{D_{m} k_{T}}{C_{S} C_{p}} \frac{\partial^{2} C}{\partial y^{2}} \\
& u \frac{\partial C}{\partial x}+v \frac{\partial C}{\partial y}=D_{m} \frac{\partial^{2} C}{\partial y^{2}}+\frac{D_{m} k_{T}}{T_{m}} \frac{\partial^{2} T}{\partial y^{2}}-k_{l}^{*}\left(C-C_{\infty}\right)
\end{aligned}
$$

The appropriate boundary conditions for the problems are given by

$$
\begin{aligned}
& \left.\begin{array}{l}
u=u_{w}(x)=c x \quad v=0 \quad T=T_{w}(x)=T_{\infty}+a x \\
C=C_{w}(x)=C_{\infty}+b x
\end{array}\right\} \text { at } y=0 \\
& u \rightarrow 0 \quad T \rightarrow T_{\infty} \quad C \rightarrow C_{\infty} \quad \text { as } y \rightarrow \infty
\end{aligned}
$$

The stretching surface has a uniform temperature $T_{w}$ and the free stream concentration is $T_{\infty}$ with $T_{w}>T_{\infty}$. Also it has a uniform concentration $C_{w}$ and the free stream temperature $C_{\infty}$ with $C_{w}>C_{\infty}$. Using the following transformations:

$$
\begin{aligned}
& \eta=\sqrt{\frac{c}{v}} y \quad u=c x f^{\prime}(\eta) v=-\sqrt{c v} f(\eta) \quad \psi=\sqrt{c v} x f(\eta) \\
& \theta(\eta)=\frac{T-T_{\infty}}{T_{w}-T_{\infty}} \phi(\eta)=\frac{C-C_{\infty}}{C_{w}-C_{\infty}}
\end{aligned}
$$

Here $\psi$ is the stream function, which is defined as

$$
u=\frac{\partial \psi}{\partial y} \quad \text { and } \quad v=-\frac{\partial \psi}{\partial x}
$$

With the help of the above relations, the governing equations finally reduce to

$$
\begin{aligned}
& \left(1+\frac{1}{\beta}\right) f^{\prime \prime \prime}+f f^{\prime \prime}-f^{2}-M f^{\prime}=0 \\
& \frac{1}{\operatorname{Pr}}(1+\varepsilon \theta) \theta^{\prime \prime}+\left(f \theta^{\prime}-f^{\prime} \theta\right)+\varepsilon \theta^{\prime}+E c\left(1+\frac{1}{\beta}\right) f^{\prime \prime}-D u \phi^{\prime \prime}=0 \\
& \frac{1}{S c} \phi^{\prime \prime}+\left(f \phi^{\prime}-f^{\prime} \phi\right)+S r \theta^{\prime \prime}-K r \phi=0
\end{aligned}
$$

The boundary conditions (5) then become

$$
\begin{array}{lrrrr}
f=0 & f^{\prime}=1 & \theta=1 & \phi=1 & \text { at } \eta=0 \\
f^{\prime} \rightarrow 0 & & \theta \rightarrow 0 & \phi \rightarrow 0 & \text { as } \eta \rightarrow \infty
\end{array}
$$

In these expressions, the prime indicates the differentiation with respect to $\eta$ and

$$
\operatorname{Pr}=\frac{k}{\mu c_{p}} \quad E c=\frac{u_{w}^{2}}{C_{P}\left(T_{w}-T_{\infty}\right)} \quad S r=\frac{D_{m} k_{T}\left(T_{w}-T_{\infty}\right)}{v T_{m}\left(C_{w}-C_{\infty}\right)}
$$




$$
M=\frac{\sigma B_{0}^{2}}{\rho c} \quad K r=\frac{k_{l}^{*}}{c} \quad S c=\frac{v}{D_{m}} \quad D u=\frac{D_{m} k_{T}}{v C_{S} C_{P}} \frac{b}{a}
$$

It is assumed that the temperature-dependent thermal conductivity $K(t)$ vary linearly with temperature Eldabe and Salwa, (1995) in the following form

$K(T)=k^{*}\left(1+\varepsilon \frac{T-T_{\infty}}{\Delta T}\right)$ in which $\alpha$ is the small parameter, $k^{*}$ is the

thermal conductivity of the fluid for away from the plate and

$\Delta T=T_{w}-T_{\infty}$. We note that $\varepsilon$ is scalar and signifies variation of the thermal conductivity with temperature.

The local skin friction coefficient $C_{f}$, the local Nusselt number $N u_{x}$ and Sherwood number $S h_{x}$ are important physical parameters for this flow heat and mass transfer situations and they given by:

The wall skin friction coefficient $C_{f}$ is defined as

$C_{f}=\frac{\tau_{w}}{\rho c^{2} x^{2}} \Rightarrow\left(\operatorname{Re}_{x}\right)^{1 / 2} C_{f}=(1+1 / \beta) f^{\prime \prime}(0)$

where $\tau_{w}=\left(\mu_{B}+\frac{p_{y}}{\sqrt{2 \pi}}\right)\left\{\frac{\partial u}{\partial y}\right\}_{y=0}$ is known as shear stress along the stretching sheet

The Nusselt number $N u_{x}$ is defined as

$N u_{x}=\frac{x q_{w}}{k\left(T_{w}-T_{\infty}\right)} \Rightarrow\left(\operatorname{Re}_{x}\right)^{-1 / 2} N u_{x}=-\theta^{\prime}(0)$

where $q_{w}=-k\left\{\frac{\partial T}{\partial y}\right\}_{y=0}$ is known as heat flux from the sheet

The Sherwood number $S h_{x}$ is defined as

$$
S h_{x}=\frac{x J_{w}}{D_{m}\left(C_{w}-C_{\infty}\right)} \Rightarrow\left(\operatorname{Re}_{x}\right)^{-1 / 2} S h_{x}=-\phi^{\prime}(0)
$$

where $J_{w}=-D_{m}\left\{\frac{\partial C}{\partial y}\right\}_{y=0}$ is known as mass flux from the sheet

where $\operatorname{Re}_{x}=\frac{x}{v} u_{w}(x)$ is the local Reynolds number

\section{NUMERICAL PROCEDURE}

Eqs. (7)-(9) are highly non-linear and hence an analytical solution does not seem to be feasible. So, these equations are solved by employing the most effective numerical shooting technique with fourth-order Runge-Kutta integration scheme (the Matlab "bvp4c" package). The utmost vital factor of this method is to choose the appropriate finite values of. $\eta \rightarrow \infty$.. In order to determine $\eta_{\infty}$ for the boundary value problem stated by equations (7)- (9), we start with some initial guess value for some particular set of physical parameters to obtain $f^{\prime \prime}(0)$. The solution procedure is repeated with another large value of $\eta_{\infty}$ until two successive values of $f^{\prime \prime}(0)$ differ only by the specified significant digit. The last vale of $\eta_{\infty}$ is finally chosen to be the most appropriate value of the limit $\eta \rightarrow \infty$ for the particular set of parameters. The value of $\eta$ may change for another set of physical parameters. Once the finite value of $\eta$ is determined then the coupled boundary value problem given by equations (7)-(9) are solved numerically using the shooting method.

In order to verify the accuracy of the present method, we have compared our results with the results of Grubka and Bobba (1985), Ishak et al. (2008) for the rate of heat transfer and rate of mass transfer. The comparisons are found to be in excellent agreement, as shown in Table 1. Therefore we are confident that our results are accurate.

\section{RESULT AND DISCUSSION}

The distributions of the velocity $f^{\prime}(\eta)$, temperature $\theta(\eta)$ and concentration $\phi(\eta)$ from equations (7)-(9), the skin friction at the surface, Nusselt and Sherwood numbers are shown in Figs. 2-9.

Table 1: Comparison of the Nusselt number for various values of $\mathrm{Pr}$ with $D u=E c=\varepsilon=0, \beta=\infty, K r=S r=0 S$

\begin{tabular}{|c|c|c|c|}
\hline \multirow{2}{*}{$\operatorname{Pr}$} & \multicolumn{3}{|c|}{ Local Nusselt Number } \\
\cline { 2 - 4 } & $\begin{array}{c}\text { Grubka and } \\
\text { Bobba, (1985) }\end{array}$ & $\begin{array}{c}\text { Ishak et al. } \\
(2008)\end{array}$ & $\begin{array}{c}\text { Present } \\
\text { study }\end{array}$ \\
\hline 1.0 & 1.0000 & 1.0000 & 1.000000 \\
3.0 & 1.9237 & 1.9237 & 1.923683 \\
10 & 3.7207 & 3.7207 & 3.720674 \\
\hline
\end{tabular}

The effect of Casson parameter $\beta$ on the velocity, temperature and concentration distributions respectively, exhibited in Figs. 2(a)-(c). It is noticed that, both the temperature and concentration fields increases with increasing values of $\beta$ and has quit opposite effect on the velocity profiles. Physically, high Casson number flows correspond to solid-like behaviour. This is due to the presence of yield stress which reduces the velocity. These results are same as noted in Swati Mukhopadhyay (2013). Also, it is noticed from Table 1 that, both rate of heat transfer and Sherwood number decrease with increasing $\beta$.

Fig. 3 illustrates the effect of magnetic parameter $M$ on the velocity profiles. The parameter $M$ measures the magnetic field strength. It can take values in the range $0<M<\infty$. The greater $M$, the stronger will be magnetic field strength. It is witnessed from the figure that the velocity field decreases with increase of $M$ along the surface. Physically, larger the Lorentz force gives the lesser velocity as this acts as a retarding force.

Figs. 4(a) and 4(b) display the influence of variable thermal conductivity parameter $\varepsilon$ on the temperature and concentration profiles respectively. It is clear that, an increase in the values of $\varepsilon$ results in an increase of temperature profile. This is due to the fact that the assumption of temperature-dependent thermal conductivity implies a reduction in the magnitude of the transverse velocity by a quantity $\frac{\partial K(T)}{\partial y}$ as can be seen from heat transfer equation (3). It is also observed that, $\varepsilon$ has opposite effect on the concentration profiles. 


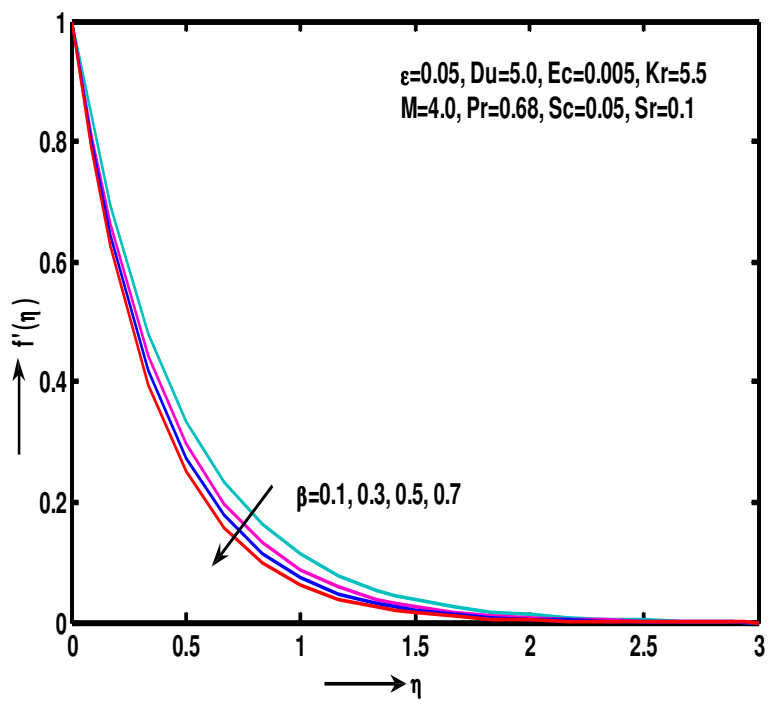

Fig. 2(a) Velocity profile for various values of $\beta$

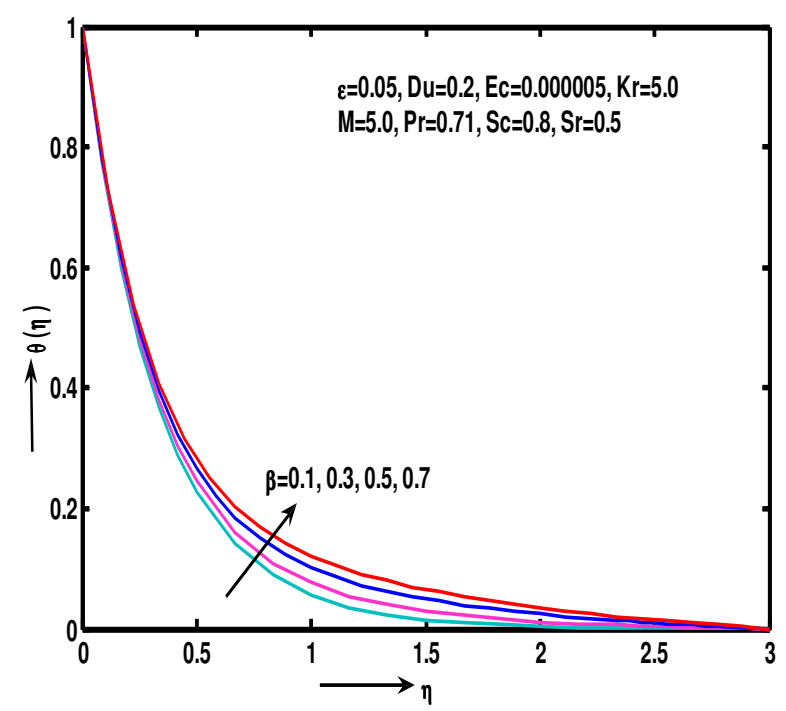

Figure 2(b) Temperature profile for various values of $\beta$

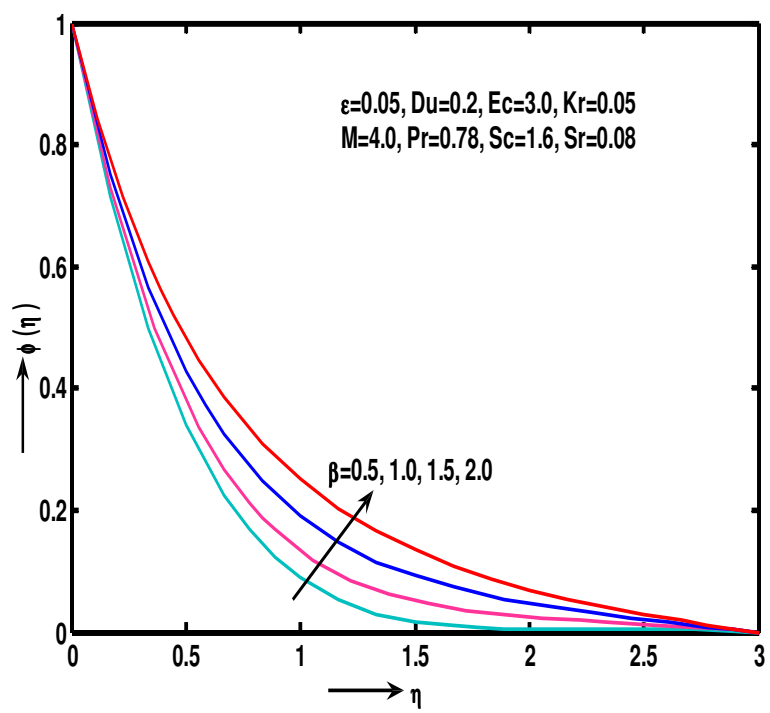

Figure 2(c) Concentration profile for various values of $\beta$

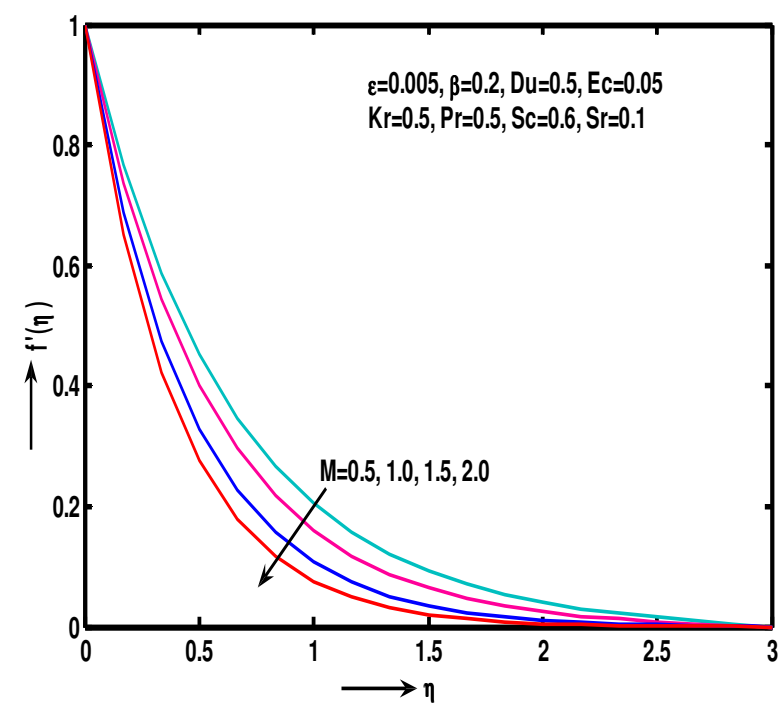

Figure 3 Velocity profile for various values of $M$

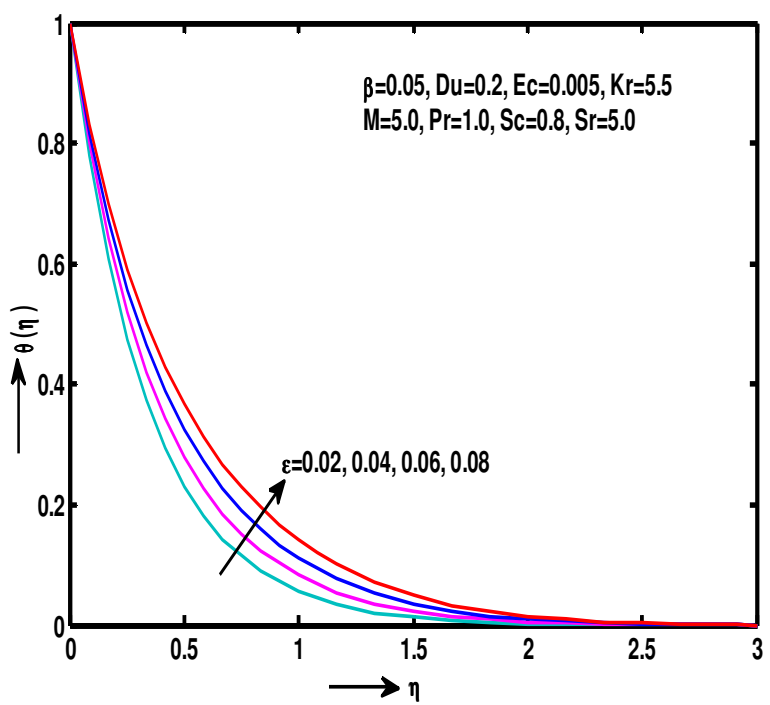

Figure 4(a) Temperature profile for various values of $\varepsilon$

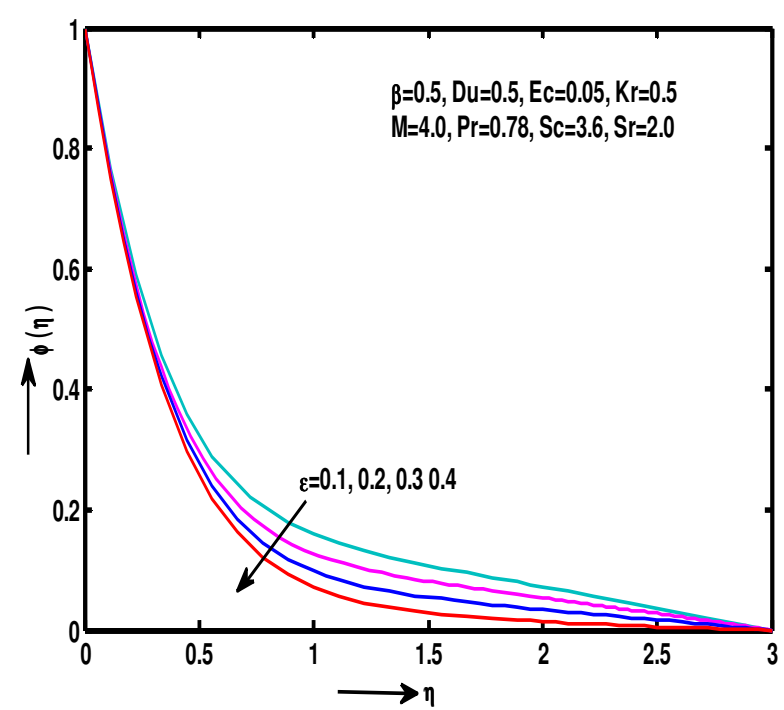

Figure 4(b) Concentration profile for various values of $\varepsilon$ 
Figs. 5(a) and 5(b) respectively, show the temperature and concentration profiles for different values of viscous dissipation parameter. The Eckert number $E c$ gives the connection between the kinetic energy in the flow and the enthalpy.

It is observed that the temperature increases with increasing $E c$ value. Physically, heat energy is deposited in the liquid due to the frictional heating. Thus the effect of increasing $E c$ is to enhance the temperature of the fluid at any point in the boundary layer. It is also observed that, $E c$ has reverse effect on the concentration profiles.

Figs. 6(a) and 6(b) display the effect of the chemical reaction parameter $\mathrm{Kr}$ on the temperature and concentration distributions respectively. It is observed that both temperature and concentration profiles decrease with the increase of chemical reaction parameter. Physically, $K r>0$, causes high molecular motion, which consequences a growth in the transport phenomenon, thereby dropping the concentration distributions in the fluid flow and matching the boundary condition on concentration as $\eta \rightarrow \infty$.

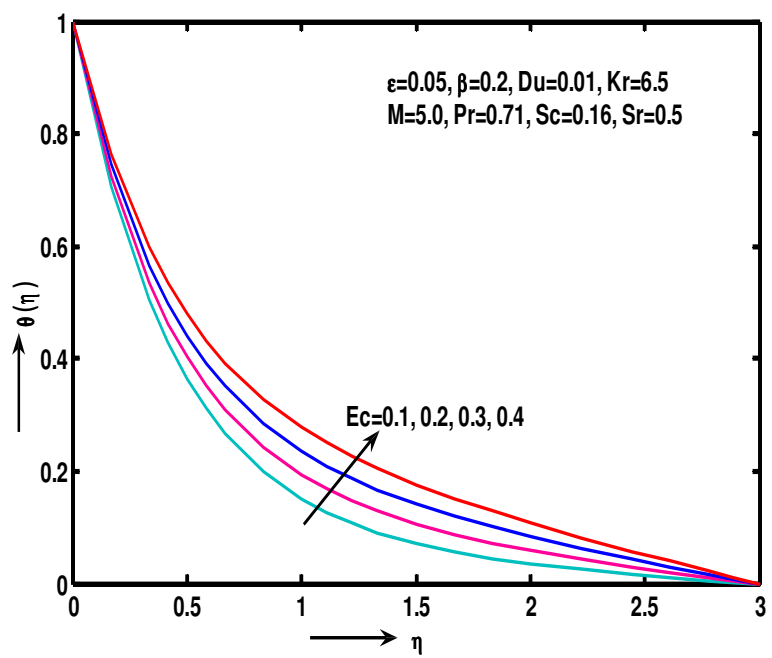

Figure 5(a) Temperature profile for various values of $E \boldsymbol{c}$

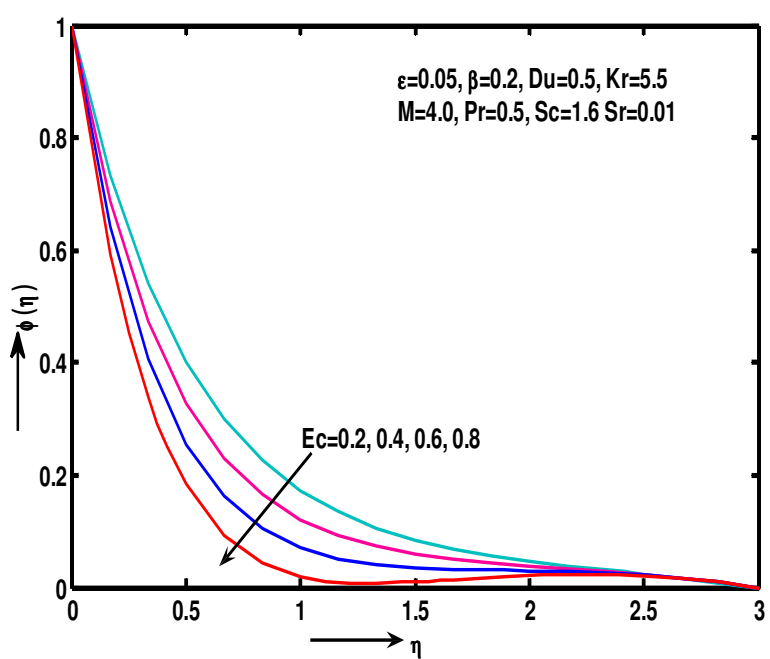

Figure 5(b) Concentration profile for various values of $E c$
Figs. 7(a) and 7(b) demonstrate the variation of temperature and concentration profiles for different values of Prandtl number $\mathrm{Pr}$. Explicitly, $P r=0.72,1.0$ and 7.0 relate to air, electrolyte solution such as salt water and water, respectively. We have randomly chosen $\operatorname{Pr}$ values to retrieve the graphical results. It is obvious that both the temperature and concentration profiles are reduced as $P r$ increase in the boundary layer. This is because larger $\operatorname{Pr}$ amounts to lesser thermal conductivity. Hence $\operatorname{Pr}$ can be used to rise the rate of cooling in conducting flows. It is also noticed from Table 1 that the local Nusselt number increases with increase of $\mathrm{Pr}$.

The effects of Soret and Dufour numbers on the temperature and concentration distributions are depicted respectively in Figs. 8(a)-8(d). It is observed that the temperature and concentration profile increase with increasing Soret number. This performance is due to the fact that Soret effect produces a mass flux from lower to higher species concentration driven by temperature gradient. On the other hand Dufour number has reverse effect on these profiles. Physically, when $D u$ is having high value then the effects of combined thermal and solutal buoyancy forces enhance convection velocity which is in turn leads to increase the temperature of the fluid by lowering the concentration of species. Hence, the Dufour effects play an important role in molecular diffusion with natural convection through a porous medium.

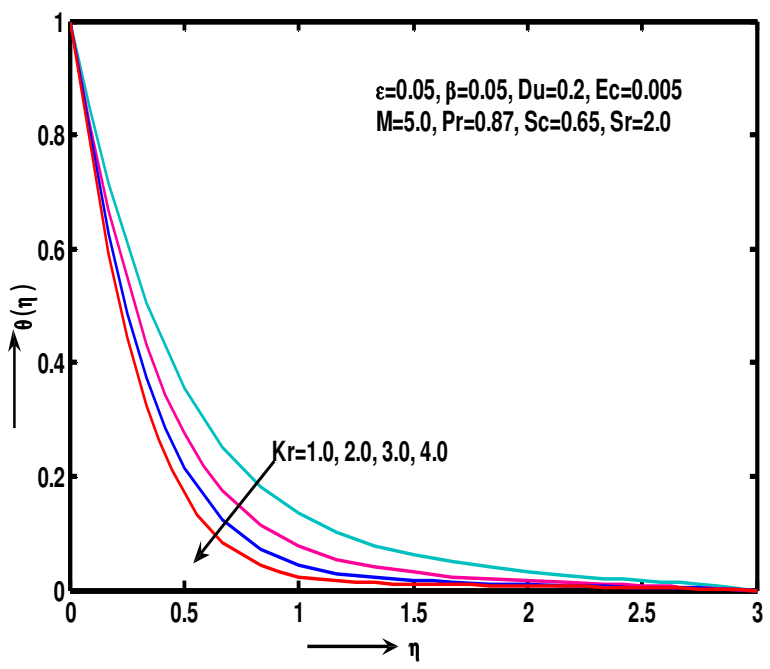

Figure 6(a) Temperature profile for various values of $\mathrm{Kr}$

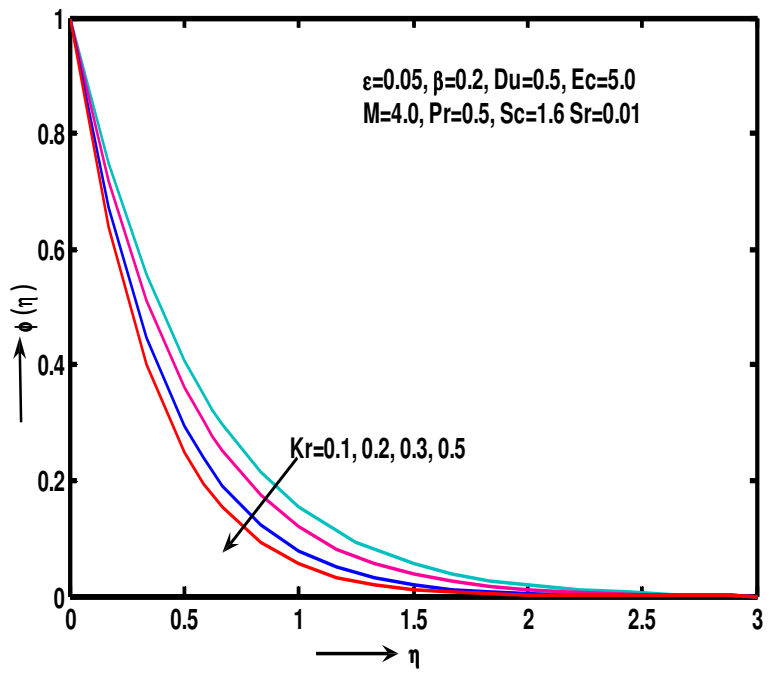

Figure 6(b) Concentration profile for various values of $\mathrm{Kr}$ 


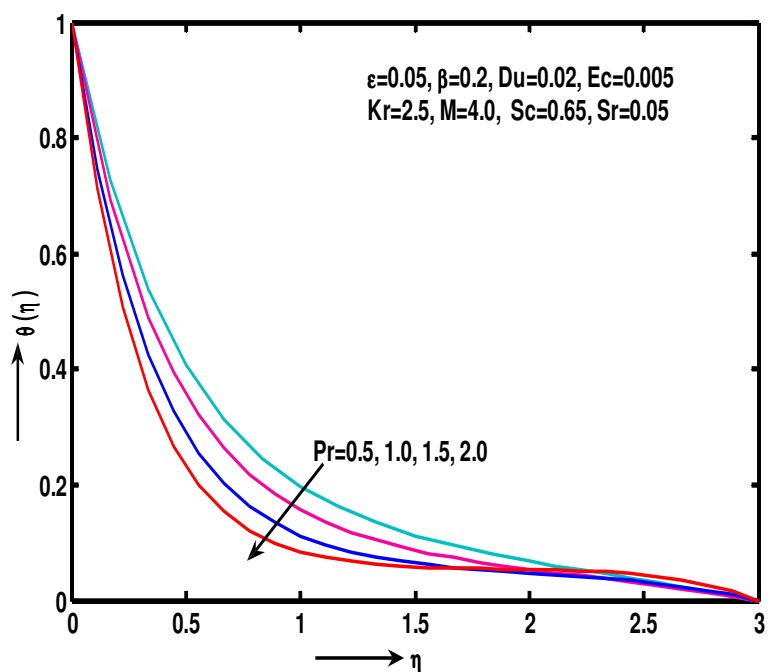

Figure 7(a) Temperature profile for various values of $\operatorname{Pr}$

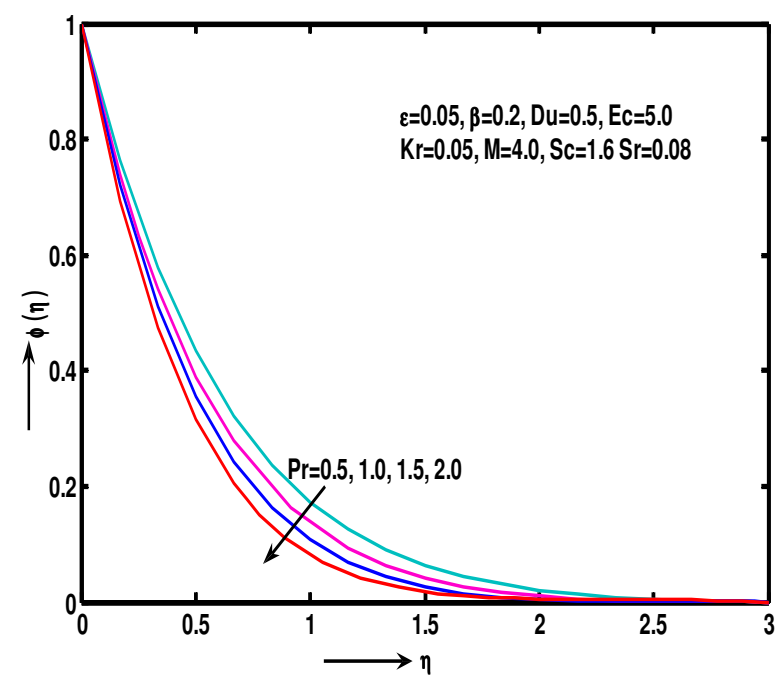

Figure 7(b) Concentration profile for various values of $\operatorname{Pr}$

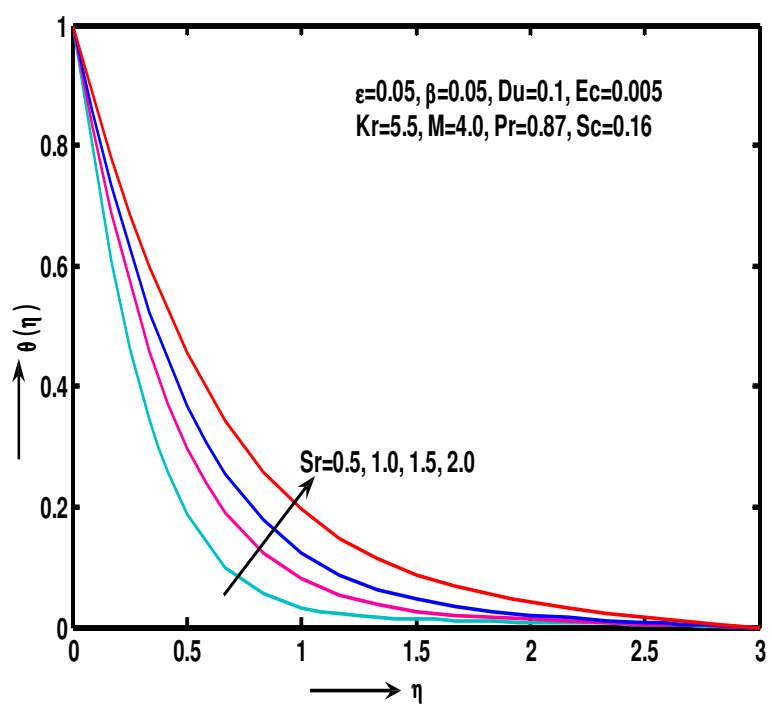

Figure 8(a) Temperature profile for various values of $S r$

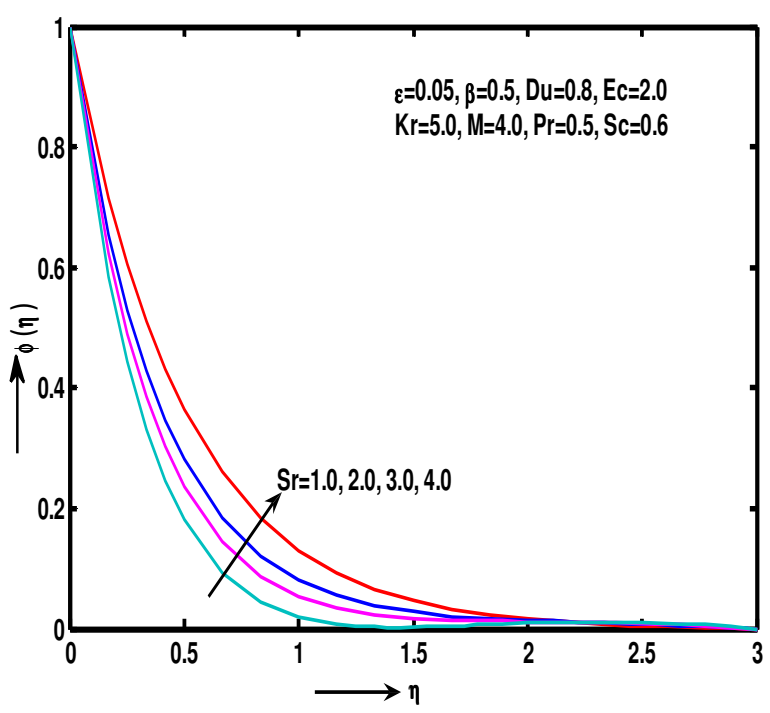

Figure 8(b) Concentration profile for various values of $\mathrm{Sr}$

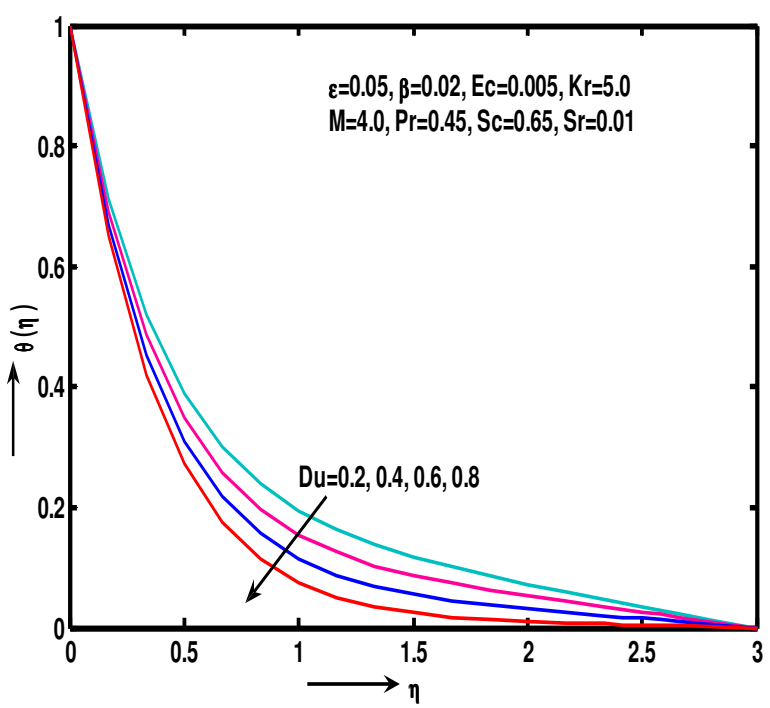

Figure 8(c) Temperature profile for various values of $\mathrm{Du}$

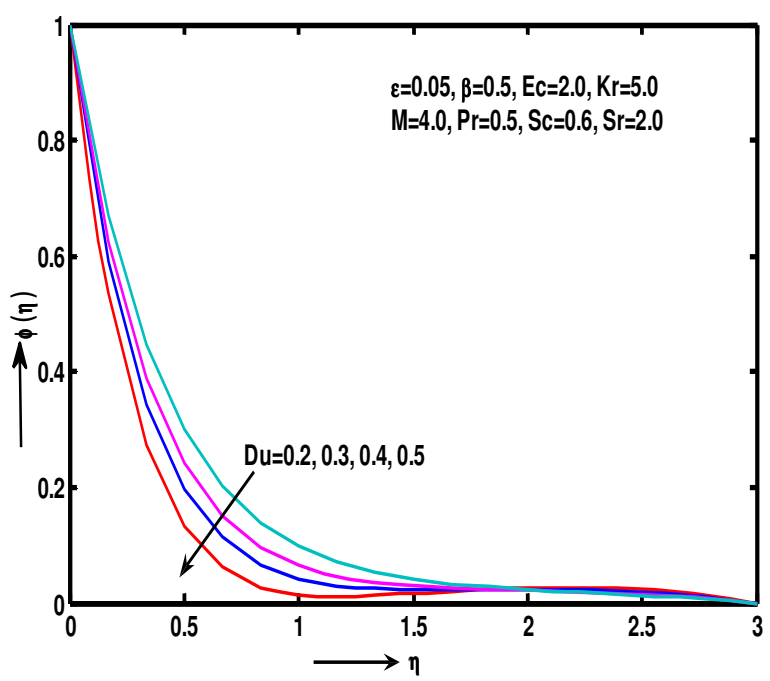

Figure 8(d) Concentration profile for various values of $\mathrm{Du}$ 
Figs. 9(a) and 9(b) depict the influence of variable thermal conductivity on the Nusselt number and the Sherwood number against thermo diffusion parameter. It is predicted that the local Nusselt number increases with an increasing of $\varepsilon$. On the other hand, the local Sherwood number decreases with increasing of $S r$. This is due to the fact that the thermal boundary layer absorbs energy which causes the temperature fall considerably with increasing the value of $S r$.

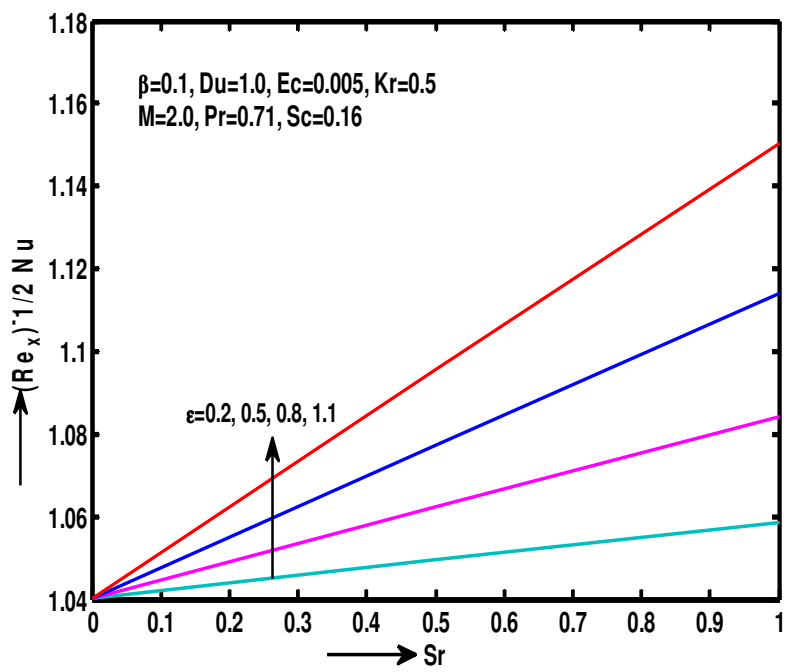

Figure 9(a) Nusselt number for various values of $\varepsilon$

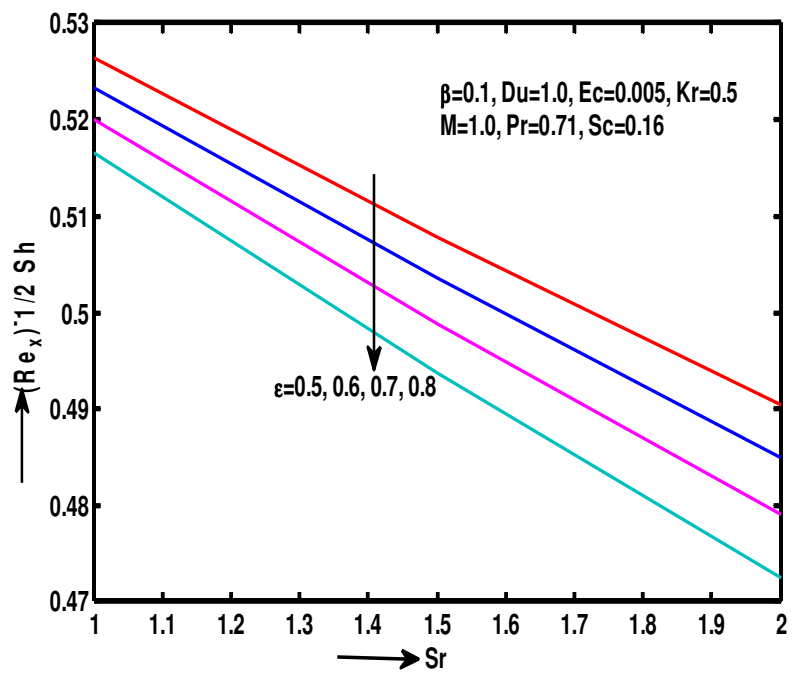

Figure 9(b) Sherwood number for various values of $\varepsilon$

\section{CONCLUSIONS}

The heat and mass transfer effects on MHD boundary layer flow of a Casson fluid model with variable thermal conductivity are addressed. The present analysis leads to the subsequent observations.

(i) The impacts of $\beta$ and $M$ on the velocity profile $f^{\prime}(\eta)$ have similar effects.

(ii) $\beta$ has opposite effects on the velocity and concentration profiles.

(iii) $\varepsilon$ has opposite effect on the temperature and concentration profiles.

(iv) Temperature increases with increase in the Eckert number and variable thermal conductivity parameter whereas reverse trend is seen with increase in the chemical reaction parameter. (v) Sherwood number decreases with increase in the value of $\varepsilon$ and $\mathrm{Sr}$ whereas reverse trend is seen on Nusselt number.

It is hoped that the results obtained will not only provide useful information for applications, but also serve as a complement to the previous studies.

\section{ACKNOWLEDGEMENTS}

The authors express their intense gratefulness to the anonymous Reviewers for their valuable comments and suggestions.

\section{NOMENCLATURE}

a positive constant

$b \quad$ positive constant

$B_{0} \quad$ constant applied magnetic field $\left(\mathrm{Wb} \mathrm{m}^{-2}\right)$

c positive constant

$C \quad$ concentration field $\left(\mathrm{kg} \mathrm{m}^{-2}\right)$

$C_{f} \quad$ skin friction coefficient

$C_{p} \quad$ specific heat at constant pressure $\left(\mathrm{J} \mathrm{kg}^{-1} \mathrm{~K}^{-1}\right)$

$C_{S} \quad$ concentration susceptibility

$C_{w} \quad$ species concentration at the wall

$C_{\infty} \quad$ uniform ambient concentration

$D_{m} \quad$ mass diffusivity

$\mathrm{Du} \quad$ Dufour number

Ec Eckert number

$e_{i j} \quad(\mathrm{i}, \mathrm{j})^{\mathrm{th}}$ component of the deformation rate

$f \quad$ dimensionless stream function

$\mathrm{Kr} \quad$ chemical reaction parameter

$k_{T} \quad$ thermal diffusion ratio

$K(T) \quad$ thermal conductivity $\left(\mathrm{Wm}^{-1} \mathrm{k}^{-1}\right)$

$k^{*} \quad$ thermal conductivity of the fluid far away from the plate

$M \quad$ magnetic field parameter

$\mathrm{Nu}$ local Nusselt number

Pr Prandtl number

$p_{y} \quad$ yield stress of the fluid

$\operatorname{Re}_{x} \quad$ local Reynolds number

$S c \quad$ Schmidt number

$\mathrm{Sr} \quad$ Soret number

Sh local Sherwood number

$T \quad$ fluid temperature (K)

$T^{4} \quad$ linear function of temperature

$T_{m} \quad$ mean temperature of the fluid (K)

$T_{w} \quad$ temperature at the wall (K)

$T_{\infty} \quad$ uniform ambient temperature (K)

$(x, y) \quad$ Cartesian coordinate's $(\mathrm{m})$

$(u, v) \quad$ velocity components along $\mathrm{x}$ and $\mathrm{y}$ axes $\left(\mathrm{ms}^{-1}\right)$

$u_{w}(x) \quad$ velocity of stretching surface $\left(\mathrm{ms}^{-1}\right)$

\section{GREEK SYMBOLS}

$\Delta T \quad$ change of variables

$\beta \quad$ Casson parameter

$v \quad$ kinematic viscosity $\left(\mathrm{m}^{2} \mathrm{~s}^{-1}\right)$

$\sigma \quad$ electrical conductivity of the fluid 
$\rho \quad$ fluid density $\left(\mathrm{kgm}^{-3}\right)$

$\varepsilon \quad$ variable thermal conductivity parameter

$\theta$ dimensionless temperature

$\phi \quad$ dimensionless concentration

$\psi \quad$ stream function $\left(\mathrm{m}^{2} \mathrm{~s}^{-1}\right)$

$\pi \quad$ product of the component of the deformation rate

$\pi_{c} \quad$ critical value of product based on the non-Newtonian model

$\mu_{B} \quad$ plastic dynamic viscosity of non-Newtonian fluids $\left(\mathrm{kgm}^{-1} \mathrm{~s}^{-1}\right)$

\section{SUBSCRIPT}

$w \quad$ condition at the wall

$\infty \quad$ condition at free stream

\section{REFERENCES}

Abd El-Aziz, M., 2007, "Temperature Dependent Viscosity and Thermal Conductivity Effects on Combined Heat and Mass Transfer in MHD Three-Dimensional Flow over a Stretching Surface with Ohmic Heating," Meccanica, 42(4), 375-386.

http://dx.doi.org/10.1016/j.jnnms.2015.07.002

Abel, M.S., Siddheshwar, P.G. and Mahesha, N., 2009, "Effects of Thermal Buoyancy and Variable Thermal Conductivity on the MHD Flow and Heat Transfer in a Power-Law Fluid Past a Vertical Stretching Sheet in the Presence of a Non-Uniform Heat Source," International Journal of Non-Linear Mechanics, 44(1), 1-12. http://dx.doi.org/10.1016/j.ijnonlinmec.2008.08.002

Animasaun, I.L., 2015, Effects of Thermophoresis, Variable Viscosity and Thermal Conductivity on Free Convective Heat and Mass Transfer of Non-Darcian MHD Dissipative Casson Fluid Flow with Suction and $\mathrm{n}^{\text {th }}$ Order of Chemical Reaction. Journal of the Nigerian Mathematical Society, 34(1), 111-31. http://dx.doi.org/10.1016/j.jnnms.2014.10.008

Animasaun, I.L., Adebile, E.A., and Fagbade, A.I., 2016, Casson Fluid Flow with Variable Thermo-Physical Property along Exponentially Stretching Sheet with Suction and Exponentially Decaying Internal Heat Generation Using the Homotopy Analysis Method, Journal of the Nigerian Mathematical Society, 35(1), 1-17. http://dx.doi.org/10.1016/j.jnnms.2015.02.001

Anjali Devi, S.P. and Prakash, M., 2015, “Temperature Dependent Viscosity and Thermal Conductivity Effects on Hydromagnetic Flow over a Slendering Stretching Sheet," Journal of the Nigerian Mathematical Society, 34(3), 318-330.

http://dx.doi.org/10.1016/j.jnnms.2015.07.002

Bhattacharya, K., Hayat, T. and Alsaedi, A., 2013, "Analytic Solution for Magneto Hydrodynamic Boundary Layer Flow of Casson Fluid over a Stretching/Shrinking Sheet with Wall Mass transfer," Chinese Physics B, 22 (2).

http://dx.doi.org/10.1088/1674-1056/22/2/024702

Bird, R.B., Dai, G.C. and Yarusso, B.J., 1983, "The Rheology and Flow of Viscoplastic Materials," Reviews in Chemical Engineering, 1, 13-15.

Casson N, 1959. A flow equation for pigment oil-suspensions of the printing ink type. Rheology of disperse systems, 84 Pergamon Press.

Eldabe, N.T.M., and Salwa, M.G.E., 1995, "Heat Transfer of MHD Non-Newtonian Casson Fluid Flow Between Two Rotating Cylinder," Journal of Phys Soc Jan, 64, 41-64.
Grubka, L.J., and Bobba, K.M., 1985, "Heat Transfer Characteristics of a Continuous, Stretching Surface with Variable Temperature," ASME J Heat Trans, 107(1), 248-250. http://dx.doi.org/10.1115/1.3247387

Hayat, T., Shehzad, S.A. and Alsaedi, A., 2012, "Soret and Dufour Effects on MHD Flow of Casson Fluid," Applied Mathematics and Mechanic-English Edition, 33(10), 1301-1312. http://dx.doi.org/10.1007/s10483-012-1623-6

Imran Ullah, Sharidan Shafie, and Ilyas Khan, 2016, "Effects of Slip Condition and Newtonian Heating on MHD Flow of Casson Fluid over a Non-Linearly Stretching Sheet Saturated in a Porous Medium," Journal of King Saud University. http://dx.doi.org/10.1016/j.jksus.2016.05.003

Ishak, R., Nazar, I. and Pop, I., 2008, "Hydromagnetic Flow and Heat Transfer Adjacent to a Stretching Vertical Sheet," Heat Mass Transfer, 44(8), 921-927.

http://dx.doi.org/10.1007/s00231-007-0322-z

Kamalakar P.V.S., Raghavender Rao R., Prasada Rao D.R.V., 2016, "Effect of chemical reaction and radiation on unsteady convective Heat and mass transfer flow of a viscous fluid in a vertical wavy Channel with oscillatory flux and heat sources," Frontiers in Heat and Mass Transfer 7(2) 1-9.

http://dx.doi.org/10.5098/hmt.7.2

Krishnendu Bhattacharyya, Uddin, M.S. and Layek, G.C., 2016, "Exact Solution for Thermal Boundary Layer in Casson Fluid Flow over Permeable Shrinking Sheet with Variable Wall Temperature and Thermal Radiation," Alexandria Engineering Journal, 55(2), 1703-1712.

http://dx.doi.org/10.1016/j.aej.2016.03.010

Kataria, H.R. and Patel, H.R., 2016, "Radiation and Chemical Reaction Effects on MHD Casson Fluid Flow Past an Oscillating Vertical Plate Embedded in Porous Medium," Alexandria Engineering Journal, 55(1), 583-595. http://dx.doi.org/10.1016/j.aej.2016.01.019

Kays, W.M., 1966, Convective Heat and Mass Transfer. McGrawHill, New York, 362.

Ling Miao and Mehrdad Massoudi, 2015, "Heat Transfer Analysis and Flow of a Slag-Type Fluid: Effects of Variable Thermal Conductivity and Viscosity," International Journal of Non-Linear Mechanics, 76, 8-19. http://dx.doi.org/1016/j.ijnonlinmec.2015.05.001

Mukhopadhyay, S., 2013, Casson Fluid Flow and Heat Transfer over a Non-Linearly Stretching Surface, Chinese Physics B, 22(7), 1-5 http://dx.doi.org/10.1088/1674-1056/22/7/074701

Mustafa, M., Hayat, T., Pop, I. and Aziz, A., 2011, Unsteady Boundary Layer Flow of a Casson Fluid Due to an Impulsively Started Moving Flat Plate, Heat Transfer, 40, 563-576 http://dx.doi.org/10.1002/htj.20358

Mustafa, M., Hayat, T., Pop, I. and Hendi, A., 2012, "StagnationPoint Flow and Heat Transfer of a Casson Fluid Towards a Stretching Sheet," Zeitschrift fur Naturforschung, 67(1-2), 70-76. http://dx.doi.org/10.5560/ZNA.2011-0057

Nadeem, S., Rizwan Ul Haq, Noreen Sher Akbar and Khan, Z.H., 2013, "MHD Three-Dimensional Casson Fluid Flow Past a Porous 
Linearly Stretching Sheet," Alexandra Engineering Journal, 52, 577-582.

http://dx.doi.org/10.1016/j.aej.2013.08.005

Olajuwon, B.I., 2013, "Effect of Thermo Diffusion and Chemical Reaction on Heat and Mass Transfer in a Power Law Fluid over a Flat Plate with Heat Generation" International Journal of Nonlinear Science, 15 (2), 117-127.

Pramanik, S., 2014, "Casson Fluid Flow and Heat Transfer Past an Exponentially Porous Stretching Surface in Presence of Thermal Radiation," Ain Shams Engineering Journal, 5, 205-212. http://dx.doi.org/10.1016/i.asej.2013.05.003

Raju, C.S.K., Sandeep, N. and Saleem, S., 2016, "Effects of Induced Magnetic Field and Homogeneous-Heterogeneous Reactions on Stagnation Flow of a Casson Fluid," Engineering Science and Technology, 19, 875-887

Raju, K.V.S., Sudhakar Reddy, T., Raju, M.C., Satya Narayana, P.V. and Venkataramana, S., 2014, "MHD Convective Flow Through Porous Medium in a Horizontal Channel with Insulated and Impermeable Bottom Wall," Ain Shams Engineering Journal, 5, 543-551.

http://dx.doi.org/10.1016/j.asej.2013.10.007

Sabir Ali Shehzad, Tasawar Hayat, Ahmed Alsaedi and Mustafa Ali Obid, 2014, "Non-Linear Thermal Radiation in Three-Dimensional Flow of Jeffrey Nanofluid: A Model for Solar Energy. Applied Mathematics and Computation, 248, 273-286.

http://dx.doi.org/10.1016/j.amc.2014.09.091

Sahin Ahmed and Karabi Kalita, 2014, "Unsteady MHD Chemically Reacting Fluid through a Porous Medium Bounded by a NonIsothermal Impulsively-Started Vertical Plate: A Numerical Technique," Journal of Naval Architecture and Marine Engineering, 11(2).

http://dx.doi.org/10.3329/jname.v11i1.10269

Saravanan, S., and Kandaswamy, P., 2000, "Low Prandtl Number Magneto Convection in Cavities: Effect of Variable Thermal Conductivity," Journal of Applied Mathematics and Mechanics, 80, 570- 576.

http://dx.doi.org/10.1016/j.jnnms.2015.07.002

Satya Narayana, P.V. and Harish Babu, D., 2015, "Numerical MHD Heat and Mass Transfer Jeffrey Fluid over a Stretching Sheet with Chemical Reaction and Radiation Parameter. Journal of the Taiwan Institute of Chemical Engineers, 59, 18-25.

http://dx.doi.org/10.1016/j.jtice.2015.07.014

Satya Narayana, P.V. and Venkateswarlu, B., 2016, "Heat and Mass Transfer on MHD Nanofluid Flow Past a Vertical Porous Plate in a Rotating System," Frontiers in Heat and Mass Transfer, 7, 8. http://dx.doi.org/10.105098/hmt.7.8
Satya Narayana, P.V., Venkateswarlu, B. and Devika, B., 2015b, "Chemical Reaction and Heat Source Effects on MHD Oscillatory Flow in an Irregular Channel" Ain Shams Engineering Journal. http://dx.doi.org/10.1016/j.asej.2015.07.012

Satya Narayana, P.V., Venkateswarlu, B. and Venkataramana, S., 2013a, "Effects of Hall Current and Radiation Absorption on MHD Micropolar Fluid in a Rotating System," Ain Shams Engineering Journal, 4(4), 843-854.

http://dx.doi.org/10.1016/j.asej.2013.02.002

Satya Narayana, P.V., Venkateswarlu, B. and Venkataramana, S., 2013b, "Chemical Reaction and Radiation Absorption Effects on MHD Micropolar Fluid Past a Vertical Porous Plate in a Rotating," Journal of Energy Heat and Mass Transfer, 35, 197-214.

Satya Narayana, P.V., Venkateswarlu, B., and Venkataramana, S., 2015a, "Thermal Radiation and Heat Source Effects on a MHD Nanofluid Past a Vertical Plate in a Rotating System with Porous Medium," Journal of Heat Transfer Asian Research, 44(1)1-19. http://dx.doi.org/10.1002/htj.21101

Sheikholeslami, M., and Rashidi, M.M., 2015, "Effect of Space Dependent Magnetic Field on Free Convection of $\mathrm{Fe}_{3} \mathrm{O}_{4}$-Water Nanofluid," Journal of the Taiwan Institute of Chemical Engineers. http://dx.doi.org/10.1016/j-jtice.2015.03.035

Swati Mukhopadhyay and Rama Subba Reddy Gorla, 2012, "Unsteady MHD Boundary Layer Flow of an Upper Convected Maxwell Fluid Past a Stretching Sheet with First Order Constructive/Destructive Chemical Reaction," Journal of Naval Architecture and Marine Engineering, 9(2). http://dx.doi.org/10.3329/jname.v9i2.12541

Talukdar, P. and Mishra, S.C., 2002, "Transient Conduction and Radiation Heat Transfer with Variable Thermal Conductivity," Numerical Heat Transfer: Part A, 41, 851-867.

http://dx.doi.org/10.1016/j.jnnms.2015.07.002

Venkateswarlu, B. and Satya Narayana, P. V., 2015, "MHD ViscoElastic Fluid Flow over a Continuously Moving Vertical Surface with Chemical Reaction," Walailak Journal of Science and Technology, 12(9).

http://dx.doi.org/10.14456/WJST.2015.54

Venkateswarlu, B. and Satya Narayana, P.V., 2015, "Chemical reaction and Radiation Absorption Effects on the Flow and Heat Transfer of a Nanofluid in a Rotating System," Applied Nano Science, 5, 351-360.

http://dx.doi.org/10.1007/s13204-014-0324-3

Vijayalakshmi A, Srinivas S, 2016, "Asymmetric flow of a nanofluid between expanding or contracting permeable walls with thermal radiation," Frontiers in Heat and Mass Transfer), 7(10) 1-11. http://dx.doi.org/10.5098/hmt.7.10 